\title{
Development of a patient-reported outcome questionnaire for aplastic anemia and paroxysmal nocturnal hemoglobinuria (PRO-AA/PNH)
}

\section{Kimmo Weisshaar}

Universitatsspital Basel https://orcid.org/0000-0001-9566-2346

Hannah Ewald

Universitatsspital Basel

Jörg P. Halter

Universitatsspital Basel

Sabine Gerull

Universitatsspital Basel

\section{Sandra Schönfeld}

Universitatsspital Basel

\section{Yuliya Senft}

Universitatsspital Basel

\section{Maria Martinez}

Universitatsspital Basel

Anne B. Leuppi-Taegtmeyer

Universitatsspital Basel

Nina Khanna

Universitatsspital Basel

Birgit Maier

Universitätsspital Basel

\section{Antonio M. Risitano}

Universita degli Studi di Napoli Federico II

\section{Regis Peffault de Latour}

Hopital Saint Louis

Andre Tichelli

Universitätsspital Basel

Jakob R. Passweg

Universitatsspital Basel

Beatrice Drexler ( $\square$ beatrice.drexler@usb.ch ) 
Keywords: aplastic anemia, paroxysmal nocturnal hemoglobinuria, patient-reported outcome, quality of life, symptom

Posted Date: March 15th, 2020

DOI: https://doi.org/10.21203/rs.3.rs-17219/v1

License: (1) This work is licensed under a Creative Commons Attribution 4.0 International License. Read Full License

Version of Record: A version of this preprint was published on September 17th, 2020. See the published version at https://doi.org/10.1186/s13023-020-01532-3. 


\section{Abstract}

Background: The introduction of new therapy modalities has significantly improved the outcome of aplastic anemia (AA) and paroxysmal nocturnal hemoglobinuria (PNH) patients. However, relatively little is known about the exact disease burden of AA/PNH since standardized assessments of symptoms including health-related quality of life (HRQoL) are frequently missing or inadequately designed for this rare patient group. We aimed to develop an $\mathrm{AA} / \mathrm{PNH}$-specific questionnaire for self-reporting of symptoms, which could be included in electronic platforms for data collection and patient care.

Methods: By scoping review, we extracted any reported symptoms in AA/PNH and their prevalence from the literature (Phase I). Consensus rounds with patients and medical experts were conducted to identify core symptoms reported in the literature and to add missing items (Phase II). Ultimately, an AA/PNHspecific patient-reported outcome (PRO) questionnaire including the selected measures was designed (Phase III).

Results: Sixty-one symptoms in AA and 45 symptoms in PNH were extracted from the literature. Twentyfour patients and seven medical experts identified 11 core symptoms including HRQoL issues after three consensus rounds. Significant differences in the symptom ranking of patients versus medical experts could be observed. We determined a compromise between experts' opinions on physical constraints and patients' frequent demands on HRQoL. These items built the basis for the development of two PRO questionnaires separate for $\mathrm{AA}$ and $\mathrm{PNH}$ following the concepts of validated instruments.

Conclusion: The development of a symptom self-reporting questionnaire for AA and PNH was feasible and the PRO questionnaire can now be validated within a web-based workflow in a subsequent feasibility study.

\section{Background}

Aplastic anemia (AA) and paroxysmal nocturnal hemoglobinuria $(\mathrm{PNH})$ are two very rare hematologic diseases [1], which can overlap or occur independently. While considerable progress has been made in the understanding of the pathophysiology and treatment of $\mathrm{AA} / \mathrm{PNH}$ in the last decades, relatively little is known about the exact disease burden on physical, mental and social health. With the implementation of the international PNH registry, clinical symptomatology and health-related quality of life (HRQoL) issues have been addressed systematically for the first time in $\mathrm{PNH}$ [2], asking for patient-reported symptoms at enrollment and documenting HRQoL by using the European Organization for Research and Treatment of Cancer Quality of Life Questionnaire (EORTC-QLQ)-C30 [3] and the Functional Assessment of Chronic IIIness Therapy Fatigue Instrument (FACIT-Fatigue) [4]. However, the latter two instruments were primarily developed for cancer patients. In AA a comparable registry does not exist, hence symptoms and HRQoL are mainly published as side observations within case reports, observational studies and interventional trials. This lack of standardized symptom assessment in AA/PNH has recently led a German expert group to develop the first disease-specific HRQoL questionnaire for AA and/or PNH [5, 6]. This marks a key step 
for improving data collection in this rare disease group. Nonetheless, the current standard method for recording symptoms includes paper forms filled out by medical staff instead of patients directly. This could lead to underreporting as symptoms are undetected by clinicians up to half the time [7]. In consequence, this approach has been questioned to be efficient, and concerns have been raised that it reflects only partially the real patient experience [8]. As an alternative method, direct reporting of symptoms by patients as 'patient-reported outcomes' (PRO) has been increasingly advocated to improve data collection, quality of care and patient outcome [9]. In particular, electronic systems offer accessible and feasible options for recording PRO (ePRO) on a regular basis by patients. Combined with a feedbacksystem to physicians this has shown to improve HRQoL and adherence to therapy, reduce admissions to the emergency department or hospital, thus leading to a better overall survival in cancer patients $[10,11]$. Based on these considerations and since systematic PRO collection has not been performed in these rare diseases so far, we saw a high potential of ePRO in AA and/or PNH. To achieve this objective, we designed the following study in three phases: First, we conducted a scoping review to collect any reported symptoms of patients with AA and/or PNH in the literature. Second, we aimed to identify core symptoms from the literature review as well as missing items by consensus rounds with medical experts and patients. In this context, we were particularly interested in measures indicating significant health deterioration and life-threat. The results of the scoping review and consensus rounds built the basis for the third phase, in which we developed a concise disease-specific PRO questionnaire for AA/PNH patients, which could be incorporated into a electronic platform for data collection and patient care in the future.

\section{Methods}

\section{Phase I (Scoping Review)}

We carried out a scoping review to provide a synthesis of patient-reported symptoms in AA/PNH. Scoping reviews yield quantified results about the knowledge available on a particular topic, differing from systematic reviews as they focus on a broader research question and less on the quality of evidence [12].

\section{Eligibility criteria}

We included patients of all ages diagnosed with either acquired AA and/or PNH. Patients with inherited bone marrow failure have been excluded. Studies reporting clinical symptoms related to AA/PNH and adverse events of commonly used AA/PNH therapies (e.g. ciclosporin, anti-thymocyte globulin, hematopoietic stem cell transplantation, eculizumab) were eligible. We did not include signs found by clinical examination (e.g. thrombosis, splenomegaly) or results of laboratory-based investigations (e.g. cytopenia, hemolysis parameters). Randomized controlled trials (RCT), observational studies as well as case reports/series were the basis for the review. We excluded animal-only studies, abstract-only publications, editorials, reviews, comments, letters, correspondences, conference abstracts and expert opinions. English and German literature published between 1980 and the 22. January 2019 was reviewed. 


\section{Identification of relevant literature}

A comprehensive scoping search on Medline and Embase via Ovid was conducted to determine the feasibility of the search. We developed the search strategy in consultation with a medical information specialist (HE) experienced in systematic reviews. Our search strategy included terms around the population and concept of PRO (Supplementary Table 1). We used text words and subject headings. Compared to Medline, Embase offered a subject heading for the term 'paroxysmal nocturnal hemoglobinuria' and retrieved more hits, hence we decided to run a full search on Embase via Ovid only. In addition, we conducted a search on Google Scholar for the detection of the most recent publications in January 2019 (Supplementary Table 2). The data search was performed between December 2018 and 22. January 2019.

\section{Data collection and analysis}

\section{Study selection}

Two reviewers (BD, KW) identified inclusion and exclusion criteria for the title/abstract- and the full-text screening (Supplementary Tables 3 and 4). The title/abstract screening and the screening of all potentially relevant full texts were done in duplicate (BD and KW) in batches of 100 and 53 references, respectively. Any disagreements regarding eligibility were resolved by joint review and discussion. When the researchers reached a consensus of at least $90 \%$, one reviewer (KW) continued the screening process of the remaining references alone.

\section{Data extraction}

One reviewer $(\mathrm{KW})$ extracted study characteristics of the included studies categorized by disease (AA, $\mathrm{PNH}, \mathrm{AA}-\mathrm{PNH}$ ) and study type (observational and RTCs, case series and reports). Symptoms related to AA and/or PNH as well as adverse events associated with AA/PNH treatment were recorded. Patient-reported symptoms related to comorbidities were not extracted. In case of similar meaning or synonymous usage, the terms were merged (e.g. hemoglobinuria and dark urine, limb pain and myalgia, dizziness and vertigo). The term 'bleeding' was further classified according to the WHO grading: minor bleeding (WHO grade 1-2) and major bleeding (WHO grade 3-4) [13]. Thereafter, all captured symptoms were put in order according to their frequency.

Since self-assessment of HRQoL in AA/PNH was limited in the literature, we supplemented the data extraction with qualitative research results obtained by Groth et al $[5,6]$. In this recent project, AA/PNH patients and physicians rated questions of the EORTC QLQ-C30 questionnaire according to their importance in $\mathrm{AA} / \mathrm{PNH}$. The highly relevant rated items were included in our data extraction. 


\section{Phase II (Consensus rounds)}

\section{Eligibility criteria}

$\mathrm{AA}$ and/or $\mathrm{PNH}$ patients as well as nurses and physicians experienced in the care of AA/PNH were eligible for the consensus rounds. Patients and medical experts required basic language skills in German or English.

\section{Recruitment}

Patients were either recruited at our institution, during an AA/PNH patient symposium in Ulm, Germany or by contact with patient advocacy groups in Germany (Aplastische Anämie \& PNH e. V. and Stiftung lichterzellen). Participating physicians and nurses were mostly from the Hematology Division of the University Hospital Basel, Switzerland. In addition, we consulted the chairperson and secretary of the Severe AA Working Party (SAAWP) of the European Bone Marrow Transplantation Society (EBMT) for their expertise.

\section{Consensus rounds using the Delphi-technique}

The literature review yielded a large number of symptoms and a selection process was required to identify the core symptoms in AA/PNH. For this purpose, we applied the modified Delphi-method, a wellestablished and previously validated system of developing consensus $[14,15]$. The technique works as a group communication process that aims to achieve a convergence of opinions on a specific matter after multiple rounds of evaluation. Three rounds have been conducted, which in previous studies have shown to be sufficient for reaching a consensus [16]

For the first two rounds, the questionnaires were sent out to the participants either by mail or e-mail. The panel was asked to review the questionnaire within four weeks and to rank the importance of the respective symptom on a Likert-type scale ranging from one to four (Importance scale: $1=$ not at all, $2=a$ little, 3 = quite a bit, 4 = very). Additionally, open feedback on missing items could be given. The questionnaires were anonymous and identified by a dedicated number for the second consensus round.

After the first and second round, the mean rating of each item was calculated. The symptom questions were additionally modified and supplemented according to the open feedback.

The first two rounds were performed with patients and experts in the above-described format. Due to divergent opinions on the main PROs between patients and experts in the first two rounds, we decided that medical experts should choose the final items in the third round since the aim of our study was the development of a questionnaire detecting life-threatening events and complications, which were less the focus of the patients' feedbacks. 
This third round was held as a round-table discussion with an extended panel of hematologists experienced in the treatment of AA/PNH patients (JRP, AT, JH, SG), in which the investigators (BD, KW) presented the results of preceding two consensus rounds. By open discussion we aimed to limit the number of symptoms that should be incorporated into the final questionnaire to max.12 items. Previous literature has shown a high practicability usage of 10 to 12 symptom items in PRO questionnaires, which could be completed quickly by the average patient (max. $20 \mathrm{~min}$ ) [17].

\section{Phase III (Design of a disease-specific PRO questionnaire)}

The core symptoms identified by the previous consensus rounds formed the basis for further development of a corresponding patient-friendly questionnaire. For this we relied on the questionnaire design used in a comparable patient population (i.e. clinical cancer research and common PRO instruments, e.g. EORTC QLQ-C30, FACIT, PRO-CTCAE) as these have demonstrated validity, reliability and sensitivity [18-20].

\section{Results}

\section{Phase I (Scoping review)}

\section{Results of the literature search and study characteristics}

The Embase search yielded 4886 records (last date of search: 8. January 2019), on Google Scholar additional 40 records were found (last date of search: 22. January 2019). On the basis of the title and abstracts 4294 records were excluded. One record could not be retrieved by the university medical library and the publisher (Pakistan Pediatric Journal) did not respond to an inquiry. A total of 619 potentially eligible records were screened in full text. Of these, 296 publications $(n=176$ for $A A, n=110$ for $P N H, n=$ 10 for AA-PNH) met our inclusion criteria and thus were used for data extraction (Figure 1).

In AA, we found one RCT, 56 observational studies and 119 case reports/series. The records for PNH included one RCT, 41 observational studies and 68 case reports/series. The AA-PNH syndrome was primarily addressed by four observational studies and six case reports/series (Supplementary Table 510).

\section{Data synthesis and analysis}

The included studies (RCTs, observational studies) comprised 12'207 individuals (AA $n=5964, \mathrm{PNH} n=$ 5016, AA-PNH $n=1227$ ). Case reports/series covered further 304 patients $(A A n=187, P N H n=110, A A-$ $\mathrm{PNH} n=7$ ). Partial overlap of the cohorts was found in seven studies of the PNH international registry or national $\mathrm{PNH}$ registries [2, 21-26], as well as in three studies by Hillmen et al. [27-29] with the SHEPHERD [30] and one of the TRIUMPH trials [31]. 
A total of 61 different symptoms could be extracted for AA, 45 symptoms for PNH and 22 for AA-PNH. The majority of symptoms in AA in the observational studies were related to cytopenia (pallor $36.6 \%$ > minor bleeding $34.5 \%$ > fever $27 \%$ > fatigue $10.2 \%$ > and dyspnea $3.5 \%$ ). In PNH patients, symptoms were most often associated to hemolysis (fatigue $41.3 \%$ > hemoglobinuria $38 \%>$ abdominal pain $30.4 \%>$ headache $18.6 \%$ > dyspnea $17.7 \%$ ). In the AA-PNH overlap patients, symptoms distinctive of both AA and PNH were reported (fatigue $58.4 \%>$ abdominal pain $40.2 \%>$ hemoglobinuria $22 \%>$ minor bleeding $21.8 \%$ $>$ dyspnea $5.5 \%$ and others $<5 \%$ ). The five most commonly reported symptoms in AA did not differ between RCT/ observational studies and case reports/series, whereas the symptoms in PNH and AA-PNH differ between observational studies/RCT and case reports/series (Figure 2 and Supplementary Table 11 and 12).

\section{Phase II (Consensus rounds)}

The extracted PRO from the literature review were incorporated into a questionnaire with closed symptom questions for AA and PNH separately, both also covering symptoms of the overlap AA-PNH syndrome. Where possible, we merged symptoms into groups (e.g. nausea, vomiting, diarrhea and constipation to gastrointestinal problems) to ease the answering process. The first AA questionnaire comprised 19 questions on clinical symptoms and 8 questions on HRQoL, whereas PNH questions focused on 22 symptoms and $8 \mathrm{HRQ}$ oL issues (Supplementary Table 13).

\section{$1^{\text {st }}$ Delphi round}

The panel of the first Delphi-round consisted of 31 participants (patients: 24, hematologists: 5 , hematology nurses: 2). Thirteen patients (mean age $54.4 \mathrm{y} ; \mathrm{f:m}=6: 7$ ) evaluated the AA questionnaire and eight (mean age $39.3 \mathrm{y} ; \mathrm{f:m}=4: 4$ ) the PNH questionnaire. Three patients (mean age $36.0 \mathrm{y} ; \mathrm{f:m}=2: 1$ ) completed both questionnaires since they were affected by either condition (Supplementary Table 14), likewise, the seven medical experts returned both questionnaires.

All items of the AA questionnaire were answered by $98 \%$ and in the PNH questionnaire by $97 \%$ of the participants. Symptoms and HRQoL questions were rated according to the predefined Likert Scale $(1=$ not at all, 2 = a little, 3 = quite a bit, 4 = very) and little open feedback was given. In the AA questionnaire, the following symptoms were stated as missing by the patients: limb cramps and spasms $(n=2)$, tremor and ataxia $(n=1)$, hand sensory dysfunction $(n=1)$. One expert suggested to additionally cover the quality of family time and availability to children. Within the PNH questionnaire, no additional item was requested. Patients and experts suggested that related items should be grouped. Symptoms covered by the AA as well the PNH questionnaire (e.g. fatigue) were overall rated similar by the two disease groups according to the mean Likert score. However, some items were judged differently between the AA and PNH patient groups: 'increased bleeding tendency', 'skin alterations', 'peripheral edema', 'limited in pursuing hobbies or other leisure time activities' as well as 'trouble taking long walks' were ranked 
remarkably higher by AA patients (difference in the Likert score $\geq 0.5$ ). 'Pain' and 'jaundice' were rated as more important by $\mathrm{PNH}$ patients (difference in Likert score $\geq 0.5$ ).

We then compared the overall experts' mean rating to the patients' mean rating. The items 'fever', 'blood pressure', 'bleeding tendency', 'dysphagia' and 'impairment in activities of daily living and work' were judged markedly higher by experts (mean difference in Likert Score: 0.7 ). 'Concentration disorder' and 'feeling irritable/tense' were considered more important by both patient groups and 'troubles in doing strenuous activities' only by AA patients (mean difference in Likert Score: 0.7) (Supplementary Table 1518).

\section{$2^{\text {nd }}$ Delphi round}

As a result of the first round the AA questionnaire was condensed to 26 and the PNH questionnaire to 27 items for the second consensus round. The overall response rate in the second round was $84.4 \%$, of which all items of both questionnaires were answered by $97 \%$ of the participants. Thirteen AA patients, five PNH patients, two AA-PNH patients and six experts rated the second questionnaire. Four patients (PNH $n=3$ and AA-PNH $n=1$ ) and one expert were lost to follow up. Again, symptoms in AA and PNH were assessed similar by patients with the exception of the items 'pain' and 'palpitations', which were rated considerably more important by PNH patients (Table 1). In return, AA patients judged the following items substantially higher than $\mathrm{PNH}$ patients (Table 1): 'increased bleeding tendency', 'tachy- or bradycardia', 'changes in hair skin or mucous membranes' and 'troubles in doing strenuous or long-lasting activities'. Comparing patients to medical experts' rating revealed again notable differences (Table 1): Experts rated the items 'fever', 'increased bleeding tendency', 'pain', 'hypertension', 'shortness of breath' and 'cough' in both AA and PNH questionnaire significantly higher (mean difference in Likert score: 1.1). In AA 'tremor/ataxia', whereas in PNH 'jaundice', 'hemoglobinuria', 'erectile dysfunction', 'dysphagia', 'gastrointestinal problems', 'perception disorder' and 'tachy- and bradycardia' were considered more important (mean difference in Likert value: 1.0). In contrast, patients rated the item 'insomnia' (mean difference in Likert value: 0.6) and the item 'mood disorder' higher. (see also Supplementary Table 19)

\section{$3^{\text {rd }}$ Delphi round}

There was a strong consensus among the experts to include the highest-rated symptoms from the previous rounds into the final questionnaire. The panel decided on the following modifications:

1) Symptoms related to hematopoietic stem cell transplantation ('changes in hair, skin and/or mucous membranes'associated to GvHD) were excluded due to the very different symptom course after transplantation and little specificity of these symptoms for AA/PNH.

2) The items 'impairment in performing strenuous activities', 'impairment in activities of daily living' and 'impaired family time' were combined with the item 'fatigue' resulting in a single question covering the 
general performance in daily life.

3) 'Palpitation'was included in the AA-PRO-questionnaire since atrial fibrillation is an important complication in the elderly, in particular with anemia [32], and there is data suggesting that AA patients could have an elevated risk [33].

4) The item 'concentration problems' was supplemented with memory dysfunction on the basis of personal experience.

5) Although most AA patients rated 'tremor and ataxia' as not relevant, the experts determined to include these items together with 'neuropathic symptoms' as well-known adverse effects of ciclosporin.

6) 'Insomnia' was not considered to be specifically related to AA or PNH and was excluded.

7) Although not rated amongst the highest in the second consensus round, the items 'dysphagia'; 'jaundice' and 'erectile dysfunction' were assessed as being particularly relevant as a marker for hemolysis in the course of PNH [34].

The final questionnaire comprised seven symptoms equally for AA and PNH (fatigue, fever, bleeding tendency, dyspnea, pain, mood and concentration/memory disorders), whereas the symptoms 'palpitations, tremors, muscle cramps and paresthesia/numbness' were only included in AA and 'dark urine, jaundice, dysphagia and erectile dysfunction' in PNH. Both questionnaires concluded with an open question to collect any further symptom the patient experienced, resulting in a total of 12 questionnaire items.

\section{Phase III (Design of the PRO AA/PNH questionnaires)}

The questions of the PRO-CTCAE (patient-reported outcome version of the Common Terminology Criteria for Adverse Events) [18] were considered most suitable for the majority of items identified by the previous consensus rounds. We also evaluated the use of questions from the EORTC-QLQ-C30 and FACIT-Fatigue, which have a main focus on HRQoL but covering less physical health and therefore seemed less appropriate for our purpose. To still address HRQoL, which was a high priority for patients, we added regularly a sub-question on the interference with daily activities (see below).

To minimize patient burden and assure greater completeness of data [17] we set-up an user-friendly structure for the questionnaire considering that patients might experience fatigue, psychosocial difficulties and time demands that make it inconvenient to answer long questionnaires. Since the PRO questionnaire is planned to be incorporated in an electronic workflow for frequently monitoring symptoms, a brief questionnaire was warranted. Based on these considerations, each symptom question was subdivided into three parts (Figure 3): 
- The first part was constructed as a 'yes/no'-question on patients' symptom experience (e.g. 'Did you feel short of breath?'). In case a patient would answer with 'no', further sub-questions would not be visible and the anwering process could be shortened significantly.

- Next the severity of the symptom (i.e. mild, moderate, severe and very severe) is addressed, following the format of the PRO-CTCAE questions.

- Third, patients are queried on how much the symptom interferes with their activities of daily living (HRQoL).

For some symptoms we decided for a modification of this design:

- We adopted the PRO-CTCAE question 'general pain' but asked for 'pain' only, by this facilitating the further query the location of pain.

- For a better characterization of 'dysphagia,' we added a question on 'alterations in eating habits/swallowing', which is necessary for CTCAE version 5.0 grading but is not part of the PROCTCAE.

- Due to the limited query of 'bleeding' in the PRO-CTCAE, we followed the modified WHO bleeding scale for locations and severity grading [13], which is highly reliable and strongly associated with platelet counts [35].

- Since the PRO-CTCAE does not cover 'fever', we formulated a question relying on our institutional definition of fever based on Petersdorf et al [36].

- To adress symptoms of 'mood disorders' (e.g. sad or unhappy feelings, anxiety, discourage) the PROCTCAE was regarded as incomplete, and instead we followed a validated two-question case-finding instrument with high sensitivity for depression by Whooley et al [37].

- None of the validated PRO questionnaires included questions on 'jaundice' and 'hemoglobinuria', therefore we phrased and graded both symptom questions based on personal clinical experience.

Table 2 shows all items included in the final questionnaires.

\section{Discussion}

This study aimed to extract all patient-reported symptoms of AA/PNH in the literature and subsequently to identify the core symptoms including HRQoL by consensus rounds with patients and medical experts. The results formed the basis for the development of a concise disease-specific PRO questionnaire for AA/PNH patients.

In the literature the most common reported symptoms addressed manifestations of pancytopenia in AA (e.g. fatigue, dyspnea, bleeding, fever) and hemolysis in PNH (e.g. abdominal pain, headache, dysphagia, erectile dysfunction). Since the standard method for documenting symptoms includes paper forms filled out by medical staff instead of patients directly, we assumed that many symptoms of AA/PNH might be underreported and the literature review is biased. In particular, data on HRQoL in AA/PNH was scarce and 
captured by questionnaires designed for cancer patients. For assessment of physical constraints rarely a standardized query was performed, emphasizing the need for AA/PNH specific tools for this matter. A first attempt to address this demand was recently made by a German group [5, 6], who developed a disease-specific HRQoL questionnaire together with patients. Our study aimed to expand this approach by designing a questionnaire not only for HRQoL but also for physical health in AA/PNH. Ultimately, the diversity of studies, including RCTs, observational studies and case reports/series made data extraction on PRO demanding since these sources were difficult to compare [38]. Accordingly, we observed differences in the frequency of recorded symptoms in case reports/series compared to observational studies and RCTs. Overall, identifying disease-specific symptoms in rare conditions in the literature is challenging and reinforced our strategy to include patients and medical experts for further identification of relevant symptoms.

Interestingly, the consensus rounds yielded only a few additional symptoms (e.g. tremor and ataxia, paresthesia, muscle spasms and cramps, impairment of family time). We assumed that our final symptom list was still not complete considering that the limited number of participating patients $(n=24)$ and medical experts $(n=7)$ cannot capture all aspects of this ultra-rare disease. This led us to add one open question in our final PRO questionnaire ("Did you have any other symptoms/problems, which were not queried above?")) to capture potential missing symptoms with this questionnaire in the future.

By including patients and medical experts in the development of the PRO questionnaire we aimed to address the needs of both parties involved. Thereby, we observed significant differences in the symptom rating by experts compared to patients: patients judged HRQoL issues (e.g. fatigue, mood disorder, impaired activities of daily living) higher whereas experts favored physical constraints (e.g. fever, bleeding, pain, dyspnea, hemoglobinuria). This finding is consistent with previous studies, where patients consider symptoms associated with their daily health status as more important and clinicians focus on unfavorable clinical outcomes (e.g. emergency room admissions, mortality) in their assessments [39]. This different perspective by patients and medical experts emphasizes the need for tools addressing both sides and educating the parties on their different needs: On the one hand, patients can be instructed in understanding symptoms more in context of their disease with relevance for life-threat [40]. On the other hand, the physicians' awareness of patients' constraints in HRQoL can be enhanced. Disease-specific tools for self-reporting of symptoms are viable options for closing these gaps, as they support individualized care of patients, can improve patient-physician communication, clinical decision making and satisfaction with care, reportedly leading to a better outcome [10,11,41,42]. However, our approach to combine patients' and experts' expectations was challenging for designing a PRO questionnaire that covers both demands while staying slim at the same time. Since AA and PNH can be life-threatening conditions, we saw a high priority for the questionnaire to detect symptoms indicating significant health deterioration and life-threat. This strategy is reinforced by the recommendations of international PNH experts to routinely monitor all PNH patients for symptoms of thromboembolic events and hemolysis [2]. To address not only factors that have value to clinicians, we also focused on HRQoL by querying impairment of daily activities and mental health. 
Up to now, PRO questionnaires have not been routinely used in the care of AA/PNH. We expect a benefit by these PRO tools since AA/PNH patients could easily report symptoms between and right before visits at specialized centers. Clinicians thereby could be informed more comprehensively on their patients' disease burden, not only at the hospital but also in-between visits by electronic transmitted PRO questionnaires, which might complement care and treatment decisions in AA/PNH. Considering that family doctors and other medical disciplines are less familiar with these ultra-rare diseases and the overwhelming and unspecific information on the internet might confuse patients, the inclusion of PRO questionnaires within web-based workflows could help to guide AA/PNH patients by detecting relevant symptoms (including side effects), providing self-management instructions, educating on the disease and documenting medication intake with electronic tools. In the setting of $\mathrm{PNH}$, this approach particularly might gain relevance as new complement inhibitors will be accessible for home administration and patients could be supported at home. For any investigator working in drug development or patientcentered outcome research this PRO instrument could also be useful for assessing symptoms and side effects in these rare conditions. Ultimately, there may also be a role for PROs in quality monitoring, which is increasingly demanded out of regulatory reasons.

Based on these considerations and results of this study, we are now planning to include our final AA/PNH-specific PRO questionnaire (Supplementary Table 20) into a web-based workflow for patient symptom-monitoring, which we intend to validate in a pursuing feasibility trial in AA/PNH patients (ClinicalTrials.gov: NCT04128943).

\section{Limitations}

We are aware of several limitations. The term 'PRO' has just recently been defined; however, the screened literature often reports on symptoms in a non-systematic manner and it was not always clear if the extracted symptoms were reported directly by patients or reflected the indirect observation of the medical team. By this, the literature review might not only have been prone to underreporting of PRO, but also may have overrepresented some symptoms in the overall frequency. In several publications a partial overlap of the population was identified (i.e. population of the international and national PNH registries, population of the TRIUMPH and SHEPHERD trial). Therefore, the extracted study population might be overestimated and symptoms overrepresented. Since the differentiation of AA, PNH and AA-PNH syndrome is not always clear-cut, the separation of symptoms according to the sub-entities was difficult for further questionnaire design. In particular, patients with an AA-PNH overlap syndrome might not be covered by our AA or PNH PRO questionnaire. Based on the low incidence of the disease, we were not able to gather a large group of patients and medical experts for the consensus rounds, which might have been needed to identify more underreported symptoms.

\section{Conclusion}


By literature review and consensus rounds with patients and medical experts it was feasible to develop a disease-specific questionnaire for self-reporting of symptoms. This PRO questionnaire can now be validated within a web-based workflow in a subsequent feasibility study in AA/PNH.

\section{Declarations}

\section{Ethics approval and consent to participate}

Not applicable

\section{Consent for publication}

Not applicable

\section{Availability of data and materials}

All data generated or analyzed during this study are included in this published article and its supplementary file

\section{Competing interests}

The authors declare that they have no competing of interest.

\section{Funding}

The project was supported by a grant from the University Basel, Switzerland, the University Hospital Basel, Switzerland and the Propatient foundation.

\section{Author contribution}

$\mathrm{KW}$ designed and performed research, analyzed data and wrote the manuscript, $\mathrm{HE}$ performed research (literature review), JPH, SG, SS, YS, MM, ABLT, NK, BM, AMR, RPL, AT, JRP performed research. BD designed and performed research, analyzed data and wrote the manuscript

\section{Acknowledgment}

The authors would like to thank all patients who participated in the consensus rounds. We are particularly grateful to Ulrike Göbel from the patient advocacy group Aplastische Anämie e.V. (www.aplastischeanaemie.de) and Pascale Burmester from the foundation for support in PNH/AA Stiftung lichterzellen (www.lichterzellen.de) who circulated the study to patients in Germany, Austria and Switzerland. We also 
thank Dr. Britta Höchsmann, who gave us the opportunity to present our project at the AA-PNH patient symposium in Ulm, Germany, and Tenzin Wangmo from the Institute for Biomedical Ethics, University Basel, Switzerland for advice on the Delphi technique.

\section{References}

1.Gulbis B, Eleftheriou A, Angastiniotis M, Ball S, Surrallés J, Castella M, Heimpel H, Hill A, Corrons JL: Epidemiology of rare anaemias in Europe. Adv Exp Med Biol2010, 686:375-396.

2.Schrezenmeier H, Muus P, Socié G, Szer J, Urbano-Ispizua A, Maciejewski JP, Brodsky RA, Bessler M, Kanakura Y, Rosse W: Baseline characteristics and disease burden in patients in the International Paroxysmal Nocturnal Hemoglobinuria Registry. Haematologica 2014, 99(5):922-929.

3.Fayers P, Bottomley A, Group EQoL: Quality of life research within the EORTC-the EORTC QLQ-C30. Eur J Cancer 2002, 38:125-133.

4.Yellen SB, Cella DF, Webster K, Blendowski C, Kaplan E: Measuring fatigue and other anemia-related symptoms with the Functional Assessment of Cancer Therapy (FACT) measurement system. J Pain Symptom Manage 1997, 13(2):63-74.

5.Groth M, S S, C N, A P-M, A R, H S, B H, T B, J P, Nameld Panse JORCIDho et al: Development of a disease-specific quality of life questionnaire for patients with aplastic anemia and/or paroxysmal nocturnal hemoglobinuria (QLQ-AA/PNH)-report on phases I and II. Ann Hematol 2017, 96(2):171-181.

6.Niedeggen C, Singer S, Groth M, Petermann-Meyer A, Röth A, Schrezenmeier H, Höchsmann B, Brümmendorf TH, Panse J: Design and development of a disease-specific quality of life tool for patients with aplastic anaemia and/or paroxysmal nocturnal haemoglobinuria (QLQ-AA/PNH)-a report on phase III. Ann Hematol 2019:1-13.

7.Pakhomov S, Jacobsen SJ, Chute CG, Roger VL: Agreement between patient-reported symptoms and their documentation in the medical record. The American journal of managed care 2008, 14(8):530.

8.Gwede C, Johnson D, Daniels S, Trotti A: Assessment of toxicity in cooperative oncology clinical trials: the long and short of it. The Journal of oncology management: the official journal of the American College of Oncology Administrators 2002, 11(2):15-21.

9. Velikova G, Booth L, Smith AB, Brown PM, Lynch P, Brown JM, Selby PJ: Measuring quality of life in routine oncology practice improves communication and patient well-being: a randomized controlled trial. J Clin Oncol 2004, 22(4):714-724.

10.Basch E, A D, M K, H S, C H, P S, L R, A B, A D, T A et al: Symptom monitoring with patient-reported outcomes during routine cancer treatment: A randomized controlled trial. J Clin Oncol2016, 34(6):557565. 
11.Basch E, Deal AM, Dueck AC, Scher HI, Kris MG, Hudis C, Schrag D: Overall Survival Results of a Trial Assessing Patient-Reported Outcomes for Symptom Monitoring During Routine Cancer TreatmentOverall Survival for Patient-Reported Symptom Monitoring in Routine Cancer TreatmentLetters. JAMA 2017, 318(2):197-198.

12.Colquhoun HL, Levac D, O'Brien KK, Straus S, Tricco AC, Perrier L, Kastner M, Moher D: Scoping reviews: time for clarity in definition, methods, and reporting. J Clin Epidemiol 2014, 67(12):1291-1294.

13.Kaufman RM, Djulbegovic B, Gernsheimer T, Kleinman S, Tinmouth AT, Capocelli KE, Cipolle MD, Cohn CS, Fung MK, Grossman BJ: Platelet transfusion: a clinical practice guideline from the AABB. Ann Intern Med 2015, 162(3):205-213.

14.Hsu C-C, Sandford BA: The Delphi technique: making sense of consensus. Practical assessment, research \& evaluation 2007, 12(10):1-8.

15.Custer RL, Scarcella JA, Stewart BR: The Modified Delphi Technique-A Rotational Modification. Journal of vocational and technical education 1999, 15(2):50-58.

16.Brooks KW: Delphi technique: Expanding applications. North Central Association Quarterly 1979, 53(3):377-385.

17.Basch E, Abernethy AP, Mullins CD, Reeve BB, Smith ML, Coons SJ, Sloan J, Wenzel K, Chauhan C, Eppard W: Recommendations for incorporating patient-reported outcomes into clinical comparative effectiveness research in adult oncology. J Clin Oncol 2012, 30(34):4249-4255.

18.Dueck AC, Mendoza TR, Mitchell SA, Reeve BB, Castro KM, Rogak LJ, Atkinson TM, Bennett AV, Denicoff AM, O'Mara AM: Validity and reliability of the US National Cancer Institute's patient-reported outcomes version of the common terminology criteria for adverse events (PRO-CTCAE). JAMA oncology 2015, 1(8):1051-1059.

19.Groenvold M, Klee MC, Sprangers MA, Aaronson NK: Validation of the EORTC QLQ-C30 quality of life questionnaire through combined qualitative and quantitative assessment of patient-observer agreement. J Clin Epidemio/ 1997, 50(4):441-450.

20.Acaster S, Dickerhoof R, DeBusk K, Bernard K, Strauss W, Allen LF: Qualitative and quantitative validation of the FACIT-fatigue scale in iron deficiency anemia. Health and quality of life outcomes 2015, 13(1):60.

21.Chou W-C, Huang W-H, Wang M-C, Chang C-S, Yeh S-P, Chiou T-J, Chen Y-C, Lin T-H, Shen M-C: Characteristics of Taiwanese patients of PNH in the international PNH registry. Thrombosis journal2016, 14(1):39.

22.Jang JH, Kim JS, Yoon S-S, Lee J-H, Kim Y-K, Jo D-Y, Chung J, Sohn SK, Lee JW: Predictive factors of mortality in population of patients with paroxysmal nocturnal hemoglobinuria (PNH): results from a 
Korean PNH registry. J Korean Med Sci 2016, 31(2):214-221.

23.Kim JS, Jang JH, Yoon S-S, Lee J-H, Kim Y-K, Jo D-Y, Chung JS, Sohn SK, Lee JW: Distinct subgroups of paroxysmal nocturnal hemoglobinuria (PNH) with cytopenia: results from South Korean National PNH Registry. Ann Hematol 2016, 95(1):125-133.

24.Patriquin CJ, Kiss T, Caplan S, Chin-Yee I, Grewal K, Grossman J, Larratt L, Marceau D, Nevill T, Sutherland DR: How we treat paroxysmal nocturnal hemoglobinuria: A consensus statement of the Canadian PNH Network and review of the national registry. Eur J Haematol 2019, 102(1):36-52.

25.Socié G, Schrezenmeier H, Muus P, Lisukov I, Roeth A, Kulasekararaj A, Lee JW, Araten D, Hill A, Brodsky R: Changing prognosis in paroxysmal nocturnal haemoglobinuria disease subcategories: an analysis of the International PNH Registry. Intern Med J 2016, 46(9):1044-1053.

26.Devos T, Meers S, Boeckx N, Gothot A, Deeren D, Chatelain B, Chatelain C, Devalet B: Diagnosis and management of PNH: Review and recommendations from a Belgian expert panel. Eur J Haematol 2018, 101(6):737-749.

27.Hillmen P, C H, J M, M E, M B, B P, M C, S R, S R, C M et al: Effect of Eculizumab on Hemolysis and Transfusion Requirements in Patients with Paroxysmal Nocturnal Hemoglobinuria. N Engl J Med 2004, 350(6):552-559.

28. Hillmen P, N Y, J S, R B, G S, P M, A R, J S, M E, R N et al: The complement inhibitor eculizumab in paroxysmal nocturnal hemoglobinuria. N Engl J Med 2006, 355(12):1233-1243.

29.Hillmen P, P M, A R, M E, A R, H S, J S, P B, J M, J S et al: Long-term safety and efficacy of sustained eculizumab treatment in patients with paroxysmal nocturnal haemoglobinuria. Br J Haematol 2013, $162(1): 62-73$.

30.Brodsky R, N Y, E A, A R, H S, J S, A G, L C, C DC, C F et al: Multicenter phase 3 study of the complement inhibitor eculizumab for the treatment of patients with paroxysmal nocturnal hemoglobinuria. Blood 2008, $111(4): 1840-1847$.

31. Hillmen P, Elebute M, Kelly R, Urbano-Ispizua A, Hill A, Rother RP, Khursigara G, Fu CL, Omine M, Browne $P$ et al: Long-term effect of the complement inhibitor eculizumab on kidney function in patients with paroxysmal nocturnal hemoglobinuria. Am J Hematol2010, 85(8):553-559.

32.Xu D, Murakoshi N, Sairenchi T, Irie F, Igarashi M, Nogami A, Tomizawa T, Yamaguchi I, Yamagishi K, Iso $\mathrm{H}$ : Anemia and reduced kidney function as risk factors for new onset of atrial fibrillation (from the Ibaraki prefectural health study). The American journal of cardiology 2015, 115(3):328-333.

33.Hu W-S, Sung F-C, Lin C-L: Aplastic Anemia and Risk of Incident Atrial Fibrillation-A Nationwide Cohort Study —. Circ J 2018, 82(5):1279-1285. 
34.DeZern AE, Brodsky RA: Paroxysmal nocturnal hemoglobinuria: a complement-mediated hemolytic anemia. Hematology/Oncology Clinics 2015, 29(3):479-494.

35.Fogarty PF, Tarantino MD, Brainsky A, Signorovitch J, Grotzinger KM: Selective validation of the WHO Bleeding Scale in patients with chronic immune thrombocytopenia. Curr Med Res Opin 2012, 28(1):7987.

36.PETERSDORF RG, BEESON PB: Fever of unexplained origin: report on 100 cases. Medicine 1961, 40(1):1-30.

37.Whooley MA, Avins AL, Miranda J, Browner WS: Case-finding instruments for depression: Two questions are as good as many. J Gen Intern Med 1997, 12(7):439-445.

38.Nguengang Wakap S, D L, A O, C R, C G, V L, D M, Y LC, A R, Nameld Rath AORCIDho et al: Estimating cumulative point prevalence of rare diseases: analysis of the Orphanet database. Eur J Hum Genet 2019:no pagination.

39.Xiao C, Polomano R, Bruner DW: Comparison between patient-reported and clinician-observed symptoms in oncology. Cancer Nurs 2013, 36(6):E1-E16.

40.Mesa RA, Miller CB, Thyne M, Mangan J, Goldberger S, Fazal S, Ma X, Wilson W, Paranagama DC, Dubinski DG: Differences in treatment goals and perception of symptom burden between patients with myeloproliferative neoplasms (MPNs) and hematologists/oncologists in the United States: Findings from the MPN Landmark survey. Cancer 2017, 123(3):449-458.

41.Shaw BE, Brazauskas R, Millard HR, Fonstad R, Flynn KE, Abernethy A, Vogel J, Petroske C, Mattila D, Drexler R: Centralized patient-reported outcome data collection in transplantation is feasible and clinically meaningful. Cancer 2017, 123(23):4687-4700.

42.Nelson EC, Eftimovska E, Lind C, Hager A, Wasson JH, Lindblad S: Patient reported outcome measures in practice. BMJ 2015, 350:g7818.

\section{Tables}

Table 1. Comparison of the mean rating (Likert scale) of questionnaire items from the second consensus round between patients and medical experts. Importance rating: $1=$ not at all, $2=$ a little, $3=$ quite a bit, 4 $=$ very. Number of participants evaluating the questionnaire: AA patients $n=13$, PNH patients $n=5$, AAPNH patients $n=2$, experts $n=6$. 
PRO-AA/PNH Questionnaire items

1. In general, do or did you feel tired?

1. Did you experience shortness of breath?

1. Do you have an increased bleeding tendency?

1. Were you limited in doing either your work or other daily as well as leisure time activities?

1. Did you have difficulties in concentrating on things?

1. Do you have any trouble doing strenuous and/or long-lasting activities?

(e.g. carrying a heavy bag, taking long walks)

1. Was your mood impaired?

$2.5 ; 2.7 \quad 3.1 ; 2.5$

(feeling depressed, being worried, feeling tense and others)

1. Did you have fever?

$1.9 ; 3.8$

$2.1 ; 3.7$

(from $38.1^{\circ} \mathrm{C}$ at least 2 times or once $\geq 38.3^{\circ} \mathrm{C}$ )

1. Did you record a high blood pressure?

$1.9 ; 2.8 \quad 1.4 ; 2.7$

(upper value $>140 \mathrm{mmHg}$, lower value $>90 \mathrm{mmHg}$ )

1. Was your sleep impaired?

$2.7 ; 2.2 \quad 2.9 ; 2.2$

(difficulties in falling asleep, staying asleep or waking up)

1. Have you been in pain?

$2.2 ; 3.2 \quad 2.7 ; 3.8$

1. Have you noticed any changes in hair, skin and/or mucous

$2.7 ; 2.7 \quad 2.1 ; 2.0$
membranes?

1. Did you feel dizzy/lightheaded/unsteady?

$2.4 ; 2.8 \quad 2.4 ; 2.5$

$2.5 ; 2.8 \quad 1.6 ; 2.2$

1. Did you record a too low or to high pulse?

( $<60$ beats/minute or $>90$ beats/minute)

1. Did you experience one or more changes in your sensory perception?

$2.0 ; 2.3$

$1.6 ; 2.5$ 
1. Did you suffer from muscle cramps/spasms?

1. Did you experience tremor ${ }^{a}$ and/or ataxia ${ }^{b}$ ?

$1.7 ; 2.5 \quad \mathrm{~N} / \mathrm{A}$

( ${ }^{a}$ uncontrolled shaking movements of the whole or parts of the body;

${ }^{\mathrm{b}}$ lack of coordination of muscle movements)

1. Was the time with your family and/or your availability to your children

$2.5 ; 2.7 \quad \mathrm{~N} / \mathrm{A}$
impaired?

1. Do you need to stay in bed or a chair during the day?

$1.9 ; 2.3 \quad 1.7 ; 2.2$

1. Did you have digestive/gastrointestinal problems?

$2.2 ; 2.3 \quad 2.1 ; 3.0$

1. Did you have a cough?

$1.7 ; 2.5 \quad 2.0 ; 2.5$

1. Did you have swelling/edema of your limbs?

$1.9 ; 2.3 \quad 1.9 ; 2.3$

1. Did you lose or gain weight unintentionally?

$2.0 ; 2.2 \quad 2.4 ; 2.3$

$1.7 ; 1.8 \quad 2.3 ; 2.3$

1. Did you experience palpitations ${ }^{c}$ ?

( ${ }^{c}$ unpleasant sensation of irregular and/or forceful beating of the heart)

1. Did your skin feel itchy?

$1.5 ; 2.0 \quad 1.6 ; 1.8$

1. Have you noticed a dark discoloration of the urine?

N/A $\quad 2.9 ; 3.8$

1. Have you noticed a yellowish discoloration of your 'white of the eye'?

N/A

$1.6 ; 3.2$

1. Men only: Do you suffer from erectile dysfunction ${ }^{d}$ ?

N/A

$1.4 ; 3.0$

( ${ }^{d}$ inability to achieve or to maintain an erection during sexual activity)

1. Did you have difficulties in swallowing things?

N/A $\quad 1.6 ; 2.7$

1. When was your last IV infusion of eculizumab?

N/A

$1.9 ; 3.3$

N/A: not applicable 
Table 2. List of final questionnaire items and source

\begin{tabular}{|c|c|}
\hline Questionnaire item & Source \\
\hline Fatigue & PRO-CTCAE Symptom Term: Fatigue \\
\hline Fever & $\begin{array}{l}\text { Center specific diagnostic and treatment guidelines based on Petersdorf et } \\
\text { al. [36] }\end{array}$ \\
\hline Bleeding & Modified WHO Bleeding Scale [13] \\
\hline Dyspnea & PRO-CTCAE Symptom Term: Shortness of breath \\
\hline Pain & According to PRO-CTCAE Symptom Term: General pain \\
\hline Mood & $\begin{array}{l}\text { According to PRO-CTCAE Symptom Term: Sad; and based on an } \\
\text { instrument by Whooley et al. [37] }\end{array}$ \\
\hline Concentration/memory & $\begin{array}{l}\text { Combination of PRO-CTCAE Symptom Term: Concentration and PRO- } \\
\text { CTCAE Symptom Term: Memory }\end{array}$ \\
\hline $\begin{array}{l}\text { Open question on other } \\
\text { symptom }\end{array}$ & N/A \\
\hline \multicolumn{2}{|l|}{$\begin{array}{l}\text { Items only for AA } \\
\text { questionnaire: }\end{array}$} \\
\hline Palpitations & PRO-CTCAE Symptom Term: Heart palpitations \\
\hline Tremor & According to CTCAE V5.0 Term: Tremor and adopted to PRO-CTCAE style \\
\hline Muscle cramps & $\begin{array}{l}\text { According to CTCAE V5.0 Term: Muscle cramp and adopted to PRO-CTCAE } \\
\text { style. }\end{array}$ \\
\hline $\begin{array}{l}\text { Paresthesia, } \\
\text { numbness }\end{array}$ & PRO-CTCAE ${ }^{T M}$ Symptom Term: Numbness \& tingling \\
\hline \multicolumn{2}{|l|}{$\begin{array}{l}\text { ltems only for } \mathrm{PNH} \\
\text { questionnaire: }\end{array}$} \\
\hline Hemoglobinuria & $\mathrm{N} / \mathrm{A}$ \\
\hline Jaundice & $\mathrm{N} / \mathrm{A}$ \\
\hline Dysphagia & $\begin{array}{l}\text { According to CTCAE V5.0 Term: Dysphagia and adopted to the PRO-CTCAE } \\
\text { Symptom Term: Difficulty swallowing }\end{array}$ \\
\hline Erectile dysfunction & PRO-CTCAE Symptom Term: Achieve and maintain erection \\
\hline
\end{tabular}

WHO: World Health Organization, PRO-CTCAE: patient-reported outcome version of the Common Terminology Criteria for Adverse Events, N/A: not applicable

\section{Figures}




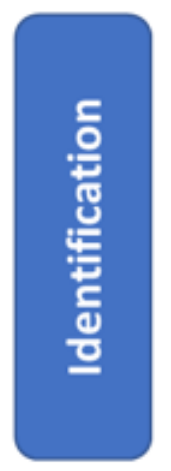

Records identified through

EMBASE (Ovid) searching

(last date of search 8. January 2019)

$(n=4886)$
Additional records identified through Google Scholar search

(last date of search 22. January 2019)

$$
(n=40)
$$
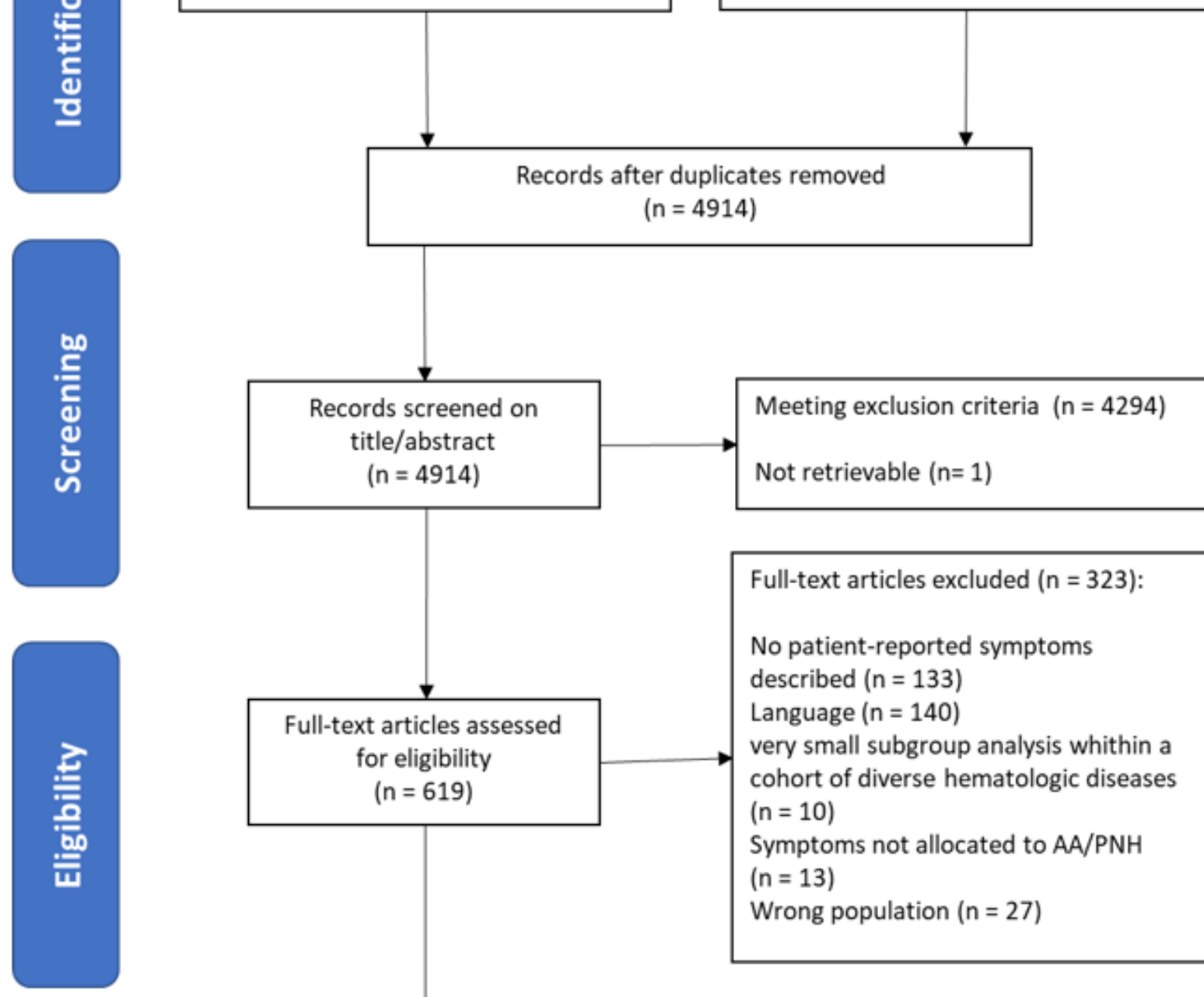

Full-text articles excluded $(n=323)$ :

No patient-reported symptoms described $(n=133)$ Language $(n=140)$ very small subgroup analysis whithin a cohort of diverse hematologic diseases ( $n=10$ )

Symptoms not allocated to AA/PNH ( $n=13$ )

Wrong population $(n=27)$

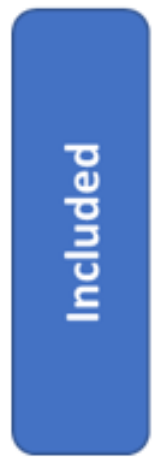

Records included for data extraction ( $n=296)$

Records referring to:

AA $(n=176)$

$\operatorname{PNH}(n=110)$

AA-PNH $(n=10)$

\section{Figure 1}

Study flow diagram for the selection of studies 


\section{A $\quad$ PRO in observational studies and RCT}

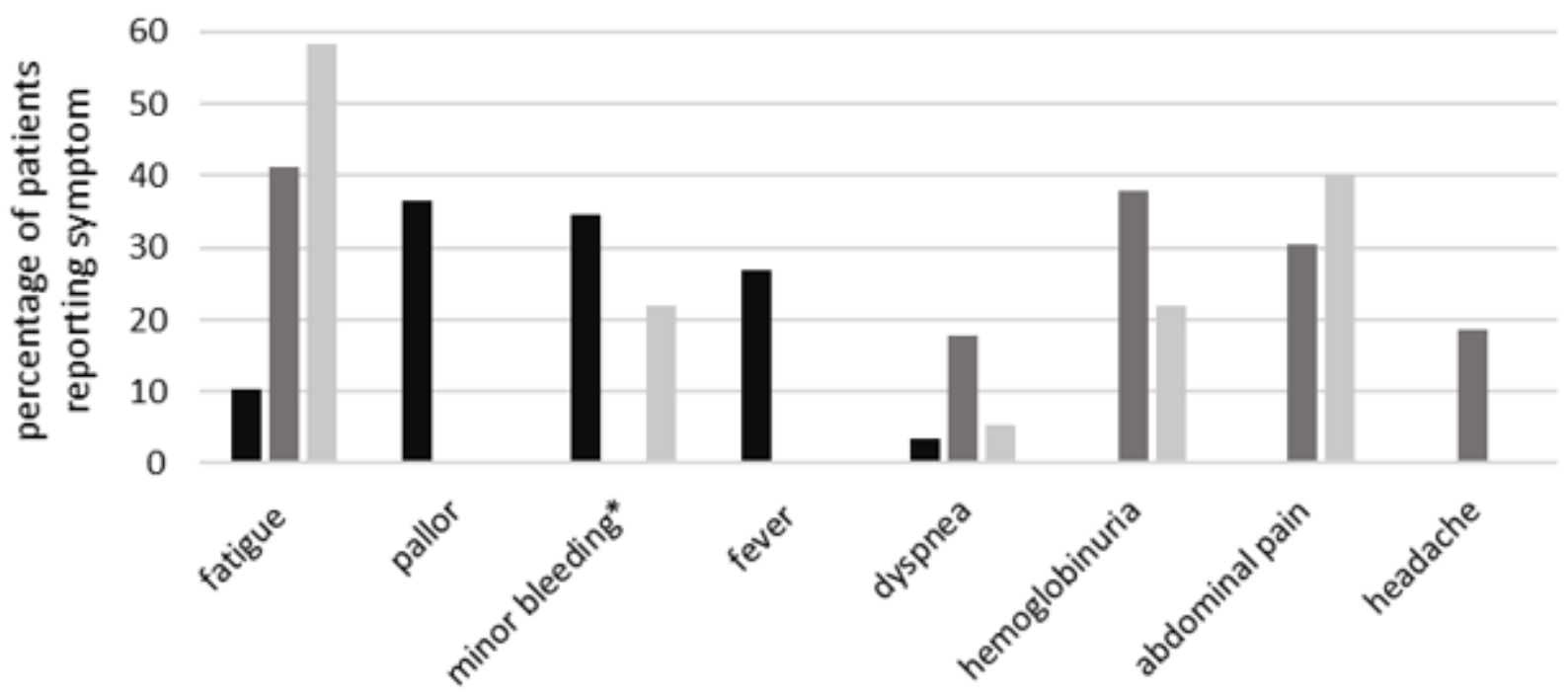

- $\mathrm{AA}(\mathrm{n}=5964) \quad$ PNH $(\mathrm{n}=5016) \quad$ AA-PNH $(\mathrm{n}=1227)$

B PRO in case reports and case series

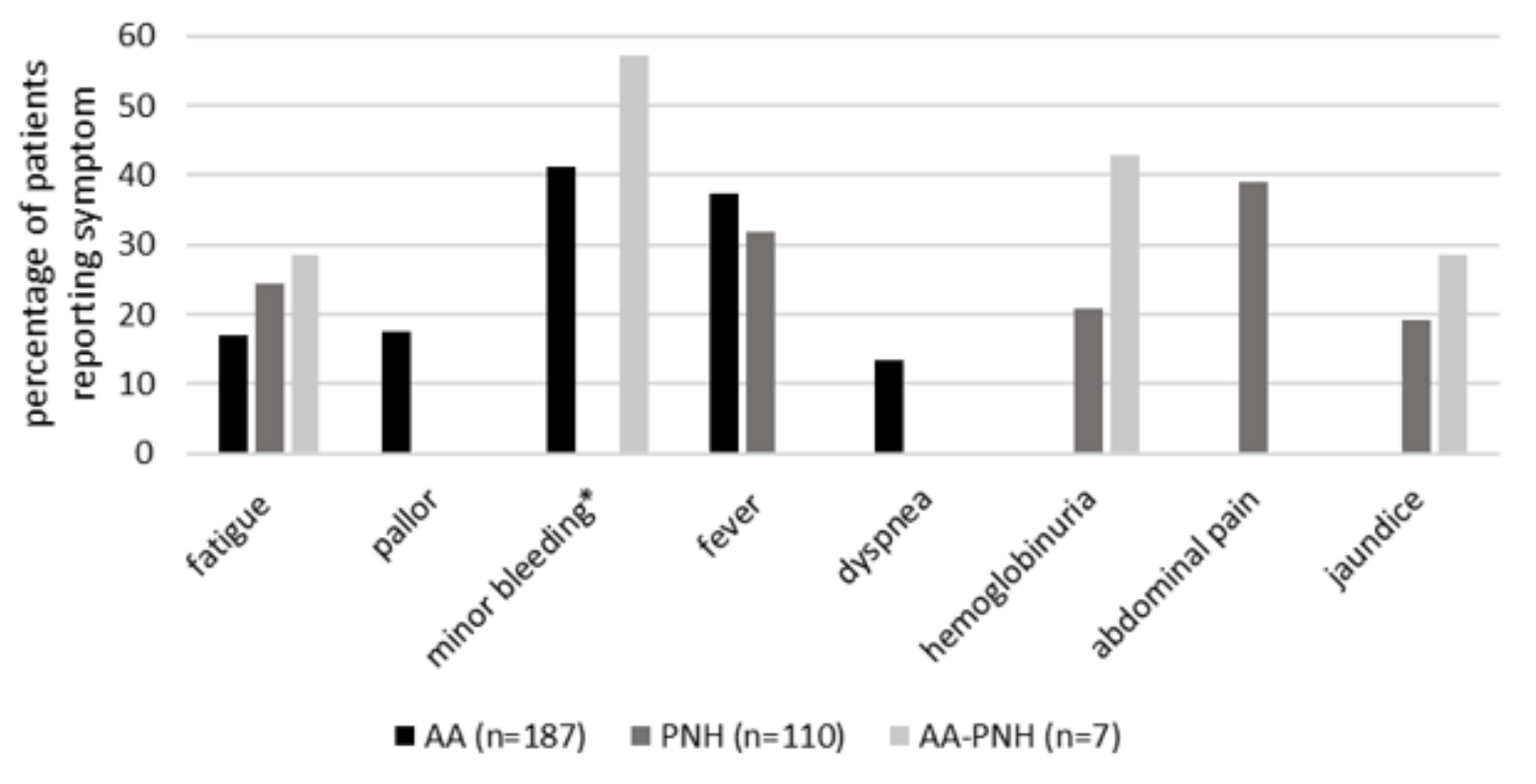

Figure 2

Patient-reported outcomes (PRO) in AA, PNH and AA-PNH in the literature. (A) Comparison of the frequency distribution of symptoms in observational studies and RCT between AA, PNH and AA-PNH. (B) Comparison of the frequency distribution of symptoms in case reports/series between $A A, P N H$ and AA$\mathrm{PNH}$. Displayed are only the five most frequently reported symptoms of each disease. *WHO bleeding grade $1-2$. 


\section{Did you feel short of breath?}

$$
\text { Yes } \bigcirc \text { no }
$$

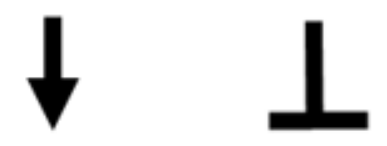

What was the severity of your shortness of breath at its worst ?

$\bigcirc$ mild

$\bigcirc$ moderate

$\bigcirc$ severe

$\bigcirc$ very severe

\section{$\downarrow$}

How much did shortness of breath interfere with your usual or daily activities?
$\bigcirc$ not at all
$\bigcirc$ a little bit
some what
$\bigcirc$ quite a bit
$\bigcirc$ very much

\section{Figure 3}

Example of a three-part symptom question.

\section{Supplementary Files}

This is a list of supplementary files associated with this preprint. Click to download. 
- SupplementarymaterialPROAAPNH2020.docx

Page 25/25 